\title{
Transfer of Skill from a Virtual Reality Trainer to Real Juggling
}

\author{
Rotem Lammfromm* ${ }^{*} \quad$ Daniel Gopher ${ }^{*}$ \\ (*)Technion - Israel Institute of Technology, Israel \\ E-mail:dgopher@ie.technion.ac.il,rotemlam@tx.technion.ac.il
}

\begin{abstract}
The purpose of this study was to evaluate transfer of training from a virtual reality environment that captures visual and temporal-spatial aspects of juggling, but not the motor demands of juggling. Transfer of skill to real juggling was examined by comparing juggling performance of novices that either experienced both the virtual training protocol and real juggling practice, or only practiced real juggling.

After ten days of training, participants who have alternated between real and virtual training demonstrated comparable performance to those who only practiced real juggling. Moreover, they adapted better to instructed changes in temporal-spatial constraints. These results imply that juggling relevant skill subcomponents can be trained in the virtual environment, and support the notion that cognitive aspects of a skill can be separately trained to enhance the acquisition of a complex perceptual-motor task.

This study was performed within the SKILLS integrated project of the EC $6^{\text {th }}$ framework.
\end{abstract}

\section{Introduction}

Juggling is a complex perceptual-motor skill, which roughly consists of three subcomponents: motor competence, memory of the ball movement pattern, and the temporal-spatial relations embedded in the execution of the pattern. The Lightweight Juggler (LWJ), developed within the SKILLS integrated project, is a two-dimensional virtual environment that can present the ball movement pattern and the temporal-spatial constraints of juggling tricks, but not the motor demands propagated by ball weight and shape. This study examines the transfer of training from the LWJ to real juggling, and examine whether the cognitive components of the skill can be separately trained in a virtual juggling environment.

Because of the temporal-spatial constraints and complexity involved in the acquisition and performance of 3-ball cascade juggling, various aspects of the task have been investigated in previous research, including learning principles [1], coordination dynamics $[2,3,4]$, visual perception $[5,6]$, and skill automatization [7]. An important representation of the spatial-temporal relations in the 3-ball cascade juggling is the dwell ratio $k$, which is defined as the "fraction of time that a hand holds on to a ball between two catches (or throws)" [8]. The $k$-ratio was shown to correlate to juggling stability and expertise, and juggling expertise can be described as the ability to restore stable juggling performance from various $k$-ratio values, and correct timing or trajectory errors $[3,8]$. The ability to explore and optimize the temporal-spatial relations of cascade juggling therefore plays a critical role in the acquisition and performance of juggling skills.

This aspect of the skill was addressed in a series of studies that aimed to explore and demonstrate the value of the Lightweight Juggler (LWJ) as a virtual trainer for the cognitive components of 3-ball cascade juggling [9]. In these studies, a combination of two virtual juggling training was shown to enhance LWJ cascade juggling: emphasis change on toss height and catch duration, and gradual speed increase. The emphasis change training protocol requires trainees to systematically allocate efforts to different sub-elements of the task $[10,11]$. When applied to training LWJ juggling skills, trainees are required to adapt their tosses to a given height or beat, which requires them to control the temporal-spatial components of the task and adapt to different $\mathrm{k}$-ratio values.

In the gradual speed increase training, task speed increases based on trainees' performance, such that initial task speed is slower than the realistic temporal constraints of the task, and increases after trainees have reached two consecutive juggling cycles in a given training block. The speed variability introduced to the task allows trainees to experience the ball movement pattern at different speeds and in particular in slowerthan-normal speeds, which enables memorization of the juggling sequence. These studies have shown that a combination of these training protocols benefits virtual juggling acquisition [9]. The LWJ virtual platform allows complete control of task properties and performance monitoring, and its low motor demands allow trainees to explore the spatial-temporal relations

This is an Open Access article distributed under the terms of the Creative Commons Attribution-Noncommercial License 3.0, which permits unrestricted use, distribution, and reproduction in any noncommercial medium, provided the original work is properly cited. 
of juggling, without the physical constraints of tossing and catching real balls.

This study aims to evaluate whether such separate training of the cognitive components of the task can transfer to enhance skill acquisition with real balls, by examining juggling performance of two groups of novices who are trained for ten days: one group is trained in real juggling only, while the other group is trained in a combination of real juggling and virtual juggling in the LWJ.

If the cognitive components of juggling can be separately trained in the virtual environment, we would expect that alternating between real juggling practice and virtual training will not interfere with juggling acquisition of beginners learning how to juggle the 3ball cascade. Furthermore, if the cognitive components of the task are effectively trained in the LWJ, not only will the virtual training not interfere with skill acquisition, but it will also enable the transfer of valuable and relevant skill components to the acquisition of cascade juggling. If this is the case, trainees who alternate between real juggling and virtual training are expected to demonstrate comparable performance to those who only practiced real juggling by the end of the training program, despite gaining less physical juggling experience, and even exhibit superior temporal-spatial task control following an effective training of these aspects of juggling.

However, if the cognitive components of juggling cannot be separately trained in the virtual environment, we expect that the performance of trainees who experience a combination of real juggling and virtual training will be inferior to that of trainees who only practice real juggling, and even suffer a decrease between real juggling sessions.

\section{Methods}

Transfer of skill was examined by training participants for ten days in both real juggling and the LWJ training protocol, and comparing their juggling performance on the $11^{\text {th }}$ day with a control group that has experienced ten days of training in real juggling. The number of consecutive juggling cycles serves as a typical index of juggling skill acquisition [12, 13].

\subsection{Participants}

Twenty-four male Technion students served as paid participants. All participants reported normal or corrected-to-normal eyesight, normal motor and hearing competence, and no prior juggling experience.

\subsection{Apparatus}

Two Polhemus Liberty ${ }^{\mathrm{TM}}$ movement tracking sensors were used in tracking hand movements in both real and virtual training conditions. Sensors were fixed to the back of participants' hands with a sports glove to maximize tracking accuracy and stability, and to their arms with a band, to minimize cord interference. Real juggling training was analyzed using the Juggling Analysis Tool (JAT) based on hand movements recorded with an Eyesweb patch, a system developed within the SKILLS integrated project. Virtual training was performed using the Lightweight Juggler platform, developed by PERCRO, which allows users to control simulated hand representations and juggle simulated balls on a two-dimensional wall-projected display. Both systems provide analysis output that includes the number of consecutive juggling cycles performed in each session. Juggling instructions were presented on the wall-projected display.

\subsection{Procedure}

Participants were randomly assigned into two groups and had ten days of training sessions and a day of test sessions, over the course of three weeks. Training sessions consisted of an instructions presentation, followed by ten four-minute training blocks, separated by two-minute breaks. The control group was trained in real juggling on all sessions, while the experimental group had 5 sessions of real juggling training and 5 sessions of virtual training on the LWJ. The training program, summarized in Table 1 , included a gradual increase in task speed on days 2 and 3, and an emphasis change protocol that emphasizes toss height, duration, or both, on days 5, 7, and 9.

Table 1. Training program for days 1-10

\begin{tabular}{|c|c|c|c|}
\hline \multirow[t]{2}{*}{ Day } & \multirow{2}{*}{$\begin{array}{l}\text { Control } \\
\text { Group }\end{array}$} & \multicolumn{2}{|c|}{ Experimental Group } \\
\hline & & $\begin{array}{l}\text { Training } \\
\text { Condition }\end{array}$ & $\begin{array}{l}\text { LWJ Training } \\
\text { Protocol }\end{array}$ \\
\hline 1 & $\begin{array}{l}\text { Real } \\
\text { Juggling }\end{array}$ & $\begin{array}{l}\text { Real } \\
\text { Juggling }\end{array}$ & \\
\hline 2 & $\begin{array}{l}\text { Real } \\
\text { Juggling }\end{array}$ & LWJ & $\begin{array}{l}\text { Gradual Speed } \\
\text { Increase }\end{array}$ \\
\hline 3 & $\begin{array}{l}\text { Real } \\
\text { Juggling }\end{array}$ & LWJ & $\begin{array}{l}\text { Gradual Speed } \\
\text { Increase }\end{array}$ \\
\hline 4 & $\begin{array}{l}\text { Real } \\
\text { Juggling }\end{array}$ & $\begin{array}{l}\text { Real } \\
\text { Juggling }\end{array}$ & \\
\hline 5 & $\begin{array}{l}\text { Real } \\
\text { Juggling }\end{array}$ & LWJ & $\begin{array}{l}\text { Emphasis Change: } \\
\text { Duration or Height }\end{array}$ \\
\hline 6 & $\begin{array}{l}\text { Real } \\
\text { Juggling }\end{array}$ & $\begin{array}{l}\text { Real } \\
\text { Juggling }\end{array}$ & \\
\hline 7 & $\begin{array}{l}\text { Real } \\
\text { Juggling }\end{array}$ & LWJ & $\begin{array}{l}\text { Emphasis Change: } \\
\text { Height or Duration }\end{array}$ \\
\hline 8 & $\begin{array}{l}\text { Real } \\
\text { Juggling }\end{array}$ & $\begin{array}{l}\text { Real } \\
\text { Juggling }\end{array}$ & \\
\hline 9 & $\begin{array}{l}\text { Real } \\
\text { Juggling }\end{array}$ & LWJ & $\begin{array}{l}\text { Emphasis Change: } \\
\text { Height and } \\
\text { Duration }\end{array}$ \\
\hline 10 & $\begin{array}{l}\text { Real } \\
\text { Juggling }\end{array}$ & $\begin{array}{l}\text { Real } \\
\text { Juggling }\end{array}$ & \\
\hline
\end{tabular}


Table 2. Day 11 conditions per block

\begin{tabular}{|c|l|}
\hline Block & \multicolumn{1}{|c|}{ Condition } \\
\hline 1 & Height: High \\
\hline 2 & Height: Low \\
\hline 3 & Height: High \\
\hline 4 & Height: Low \\
\hline 5 & Rhythm: Fast \\
\hline 6 & Rhythm: Slow \\
\hline 7 & Rhythm: Fast \\
\hline 8 & Rhythm: Slow \\
\hline 9 & No adaptation \\
\hline 10 & No adaptation \\
\hline
\end{tabular}

On day 11 , both groups were instructed to adapt to height and rhythm changes during real juggling, as listed in Table 2. During the Height conditions, they were instructed to juggle lower than normal or higher than normal, and during the Rhythm conditions they were instructed to juggle according to a given pace.

\section{Results}

The mean daily records of consecutive juggling cycles for the experimental group and the control group is presented in Figure 1, for days in which they trained in real juggling.

Technical limitations of our recording and analysis tools currently prevent an accurate analysis of slowpaced juggling. Therefore, of the four temporal-spatial adaptation conditions on day 11 , we were only able to generate a valid analysis of juggling activity for the Fast Rhythm condition. Figure 2 depicts the mean record for each group in the real juggling task on days 10 and 11 without temporal-spatial adaptation requirements, and under the Fast Rhythm condition on day 11 .

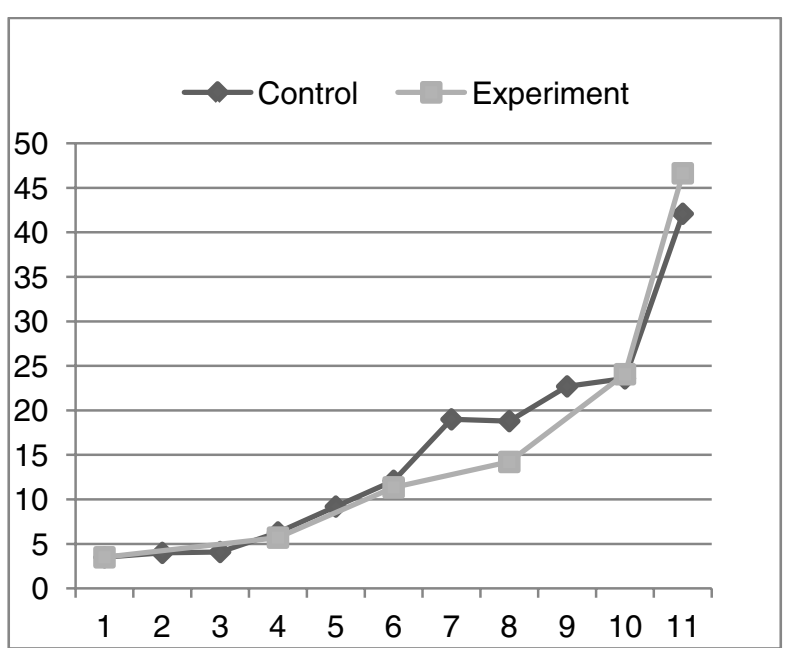

Fig. 1. Mean daily record of consecutive juggling cycles per group per day.

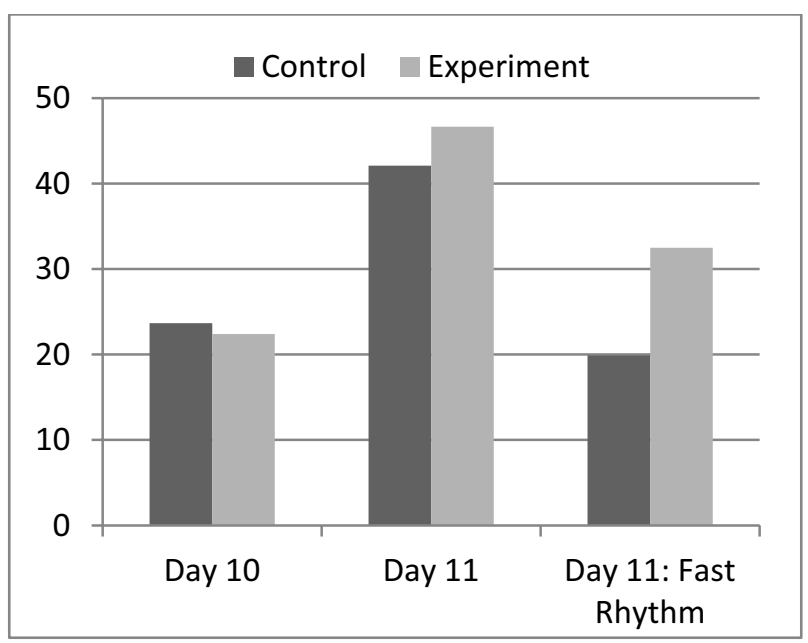

Fig. 2. Mean record of consecutive juggling cycles per group on days 10 and 11, and under the fast rhythm adaptation condition on day 11 .

\section{Discussion}

This study follows a series of studies that explore the value of cognitive training in a virtual juggling environment, as a case study for the acquisition of a complex skill [9]. Our findings provide initial evidence for transfer of skill from the LWJ to real juggling: juggling performance with real balls was equal in alternated training and exclusive real balls training conditions. Moreover, as Figure 1 shows, juggling performance in the alternated training conditions progressed smoothly and monotonically despite the 6 transitions from training in the LWJ and juggling with real balls. By the end of the training program, participants in both groups reach a similar level of competence in the 3-ball cascade task, and the experimental group had a certain advantage over the control group in a task that requires adaptation to the temporal-spatial constraints imposed by a fast rhythm, as Figure 2 shows. These findings imply that the training protocol implemented in the LWJ contributes to relevant skill subcomponents, despite the low motor and sensory fidelity of the virtual environment. More generally, they support the notion that the cognitive aspects of skill can be separately trained in a virtual environment to enhance the acquisition of a complex perceptual-motor task.

Future research could shed more light on the nature of cognitive juggling training in the LWJ and its contribution to skill acquisition. Cognitive-based virtual environments and training protocols may be examined for training other complex motor skills. A 
cognitively-effective training protocol implemented in a low-fidelity virtual environment could benefit various skills in the context of athletic, technical or defense training, while providing a fully controlled, monitored and customizable training environment, and often a low-cost and low-risk replacement for the real task environment.

\section{References}

[1] E. Trussell. Prediction of success in motor skill on the basis of early learning achievement, Research Quarterly, 36, 342-347, 1965.

[2] P.J. Beek. Juggling dynamics, Amsterdam: Free University Press, 1989.

[3] P.J. Beek, and M.T. Turvey. Temporal patterning in cascade juggling, Journal of Experimental Psychology: Human Perception and Performance, 18, 934-947, 1992.

[4] A.A. Post, A. Daffertshofer, and P.J. Beek. Principal components in three-ball cascade juggling, Biological Cybernetics, 82, 143-152, 2000.

[5] A.A.M. Van Santvoord and P.J. Beek. Phasing and the pick-up of optical information in cascade juggling, Ecological Psychology, 6, 239-263, 1994.

[6] R. Huys and P.J. Beek. The coupling between point-ofgaze and ball movements in three-ball cascade juggling: The effects of expertise, pattern and tempo, Journal of Sports Sciences, 20, 171-186, 2002.

[7] J.M. Bebko, J.L. Demark, P.A. Osborn, S. Majumder, C.J. Ricciuti, and T. Rhee. Acquisition and automatization of a complex task: An examination of three-ball cascade juggling, Journal of Motor Behavior, $35,109-118,2003$.

[8] P.J. Beek and A. Lewbel. The science of juggling, Scientific American, 273(5), 92-97, 1995.

[9] J. Lagarde, V. Erev, V. Lippi, G. Zelic, C.A. Avizzano, and D. Gopher. Training to juggle with a light weight juggler. In M. Bergamasco, B.G. Bardy, and D. Gopher, Skills training in multimodal virtual environments, Taylor \& Francis, in press.

[10] D. Gopher, M. Weil, and T. Bareket. Transfer of skill from a computer game trainer to flight, Human Factors, 36, 1-19, 1994.

[11] D. Gopher, M. Weil, and D. Siegel. Practice under changing priorities: An approach to training of complex skills, Acta Psychologica, 71, 147-179, 1989.

[12] C. Knapp and W.R. Dixon. Learning to juggle: I. A study to determine the effect of two different distributions of practice on learning efficiency, The Research Quarterly, 21, 331-336, 1950.

[13] C. Knapp and W.R. Dixon. Learning to juggle: II. A study of whole and part methods, The Research Quarterly, 23, 398-401, 1952. 